\title{
Das Zusammenspiel des Teams ist entscheidend
}

Jährlich werden deutschlandweit ca. 1 Million Implantate gesetzt (nach der DGI). Entscheidet sich ein Patient gemeinsam mit dem Zahnarzt für eine hochwertige Implantat-Versorgung, ist eine ausführliche Beratung, Diagnostik, Vorbehandlung und Nachsorge nötig.

Unser Ziel muss es sein, die Patienten mit langlebigem, gutem Zahnersatz zu versorgen und das Risiko periimplantärer Erkrankungen zu minimieren. Die Basis dafür sind neben dem Fachwissen eine gute Aufklärungskompetenz, ein spezielles Prophylaxe-Konzept für bereits mit Implantaten versorgte Patienten, eine gesicherte intraorale Hygienefähigkeit, spezielle Hilfsmittel zur individuellen Mundhygiene sowie die Compliance des Patienten. Zusammen mit dem Zahnarzt, der ProphylaxeFachkraft sowie den Praxismitarbeitern spielen der Patient und seine Bereitschaft zur Mitarbeit eine wesentliche Rolle.

Der mit Implantaten versorgte Patient hat für mich als praktisch tätige Dentalhygienikerin immer ein erhöhtes Risiko, an einer Periimplantitis zu erkranken. Eine regelmäßige, professionelle Kontrolle, Nachsorge und Implantat-Prophylaxe in einem Zeitraum von mindestens 3 bis 4 Monaten ist daher zu empfehlen. Hier fehlen jedoch klar definierte Nachsorge-Standards.

\section{Möglichkeiten und Grenzen der Implantat-Pflege}

Implantat-Prophylaxe erfordert Ausdauer des Patienten, aber auch des Praxisteams. Das zeigt das Beispiel eines 60-Jährigen, der seit 7 Jahren mit einem Implantat Regio 15 versorgt ist. Er kommt vierteljähr-

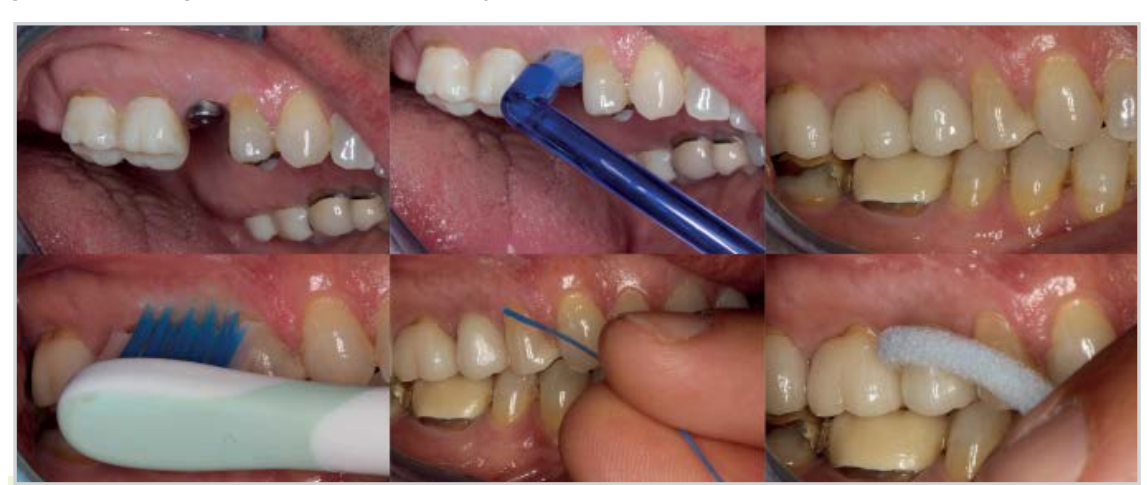

Positive Entwicklung einer Implantat-Prophylaxe

Gesamter Behandlungsablauf und Implantat-Nachsorge über den Zeitraum einiger Jahre, Behandlungsschritt und Materialien je Zeile v.I.n.r.: Implantat nach der Operation und Freilegung, Einheilschraube, ohne Suprakonstruktion; Mechanische Reinigung mit Einbündelbürste (soft) und meridol Mundspüllösung für einige Tage; Implantat-Situation mit Supraversorgung (Krone) nach 7 Jahren; Nachsorge alle 3-4 Monate; Regelmäßiges professionelles Implantat-Cleaning, mit Luft-Pulver-Wasserstrahlgerät, mit anschließender intraoraler Mundhygieneanweisung zur häuslichen Plaque-Kontrolle; Hier: Mundhygiene-Instruktion, Demonstration meridol ${ }^{\circledR}$ Floss und elmex ${ }^{\circledR}$ Zahnbürste (elektrisch) (Bild: Sabrina Dogan).

lich zur Implantat-Nachsorge in unsere Praxis. Das Implantat ist entzündungsfrei, es ist kein Biofilm vorhanden, der Bleeding-on-Probing-Test (Bluten auf Sondieren) ist negativ, die Sondierungstiefen sind zirkulär, an 6 Stellen des Implantats mittels Kunststoffsonde mit 3 mm bemessen. An Nachbarzähnen sowie an vorhandenen Restaurationen sind leichte weiche Zahnbeläge sichtbar, die Sondierungstiefen bemessen generell 2-3 mm.

\section{Die alternde Gesellschaft als Maßstab für eine professionelle Periimplantitits-Prävention}

Die alternde Gesellschaft stellt neue Herausforderungen an zahnärztliche Praxen und Pflegepersonal im Zusammenhang mit der Implantat-Pflege. Leidet beispielsweise ein Implantat-Patient plötzlich an einschränkenden Krankheit, kann adäquate Mundhygiene in der Regel nicht mehr umgesetzt werden. Gleiches gilt für längere stationäre Behandlungen. Die Gefahr von irreversiblen Krankheitsbildern in der Mundhöhle steigt. Generell kommt es zukünftig mehr denn je auf eine gute Implantat-Prophylaxe für alle Patienten an. Um diese zu gewährleisten, ist neben der interdisziplinären Zusammenarbeit mehrerer Fachgruppen auch die 